\title{
Neuropsychological effects of exposure to naphtha among automotive workers
}

\author{
Roberta F White, Thomas G Robins, Susan Proctor, Diana Echeverria, Adrian S Rocskay
}

\begin{abstract}
The association between exposure to naphtha and neurobehavioural measures was examined prospectively over one year among workers employed at an automotive plant that used naphtha to calibrate fuel injectors. The neurobehavioural tests included those that assess mood, basic intelligence, and functioning of the cerebral frontal lobes and limbic system and were designed so that acute, reversible, and chronic effects of solvent exposure could be assessed. Participants were 248 workers in June 1988, and the testing was repeated on 185 of these workers in 1989. Concentrations of naphtha at the plant ranged from six to $709 \mathrm{mg} / \mathrm{m}^{3}$, although exposure was greater in 1988 than in 1989. Duration of exposure for individual subjects ranged from 0.8 to 7.3 years. Cross sectional data analyses showed significant associations between level of exposure to naphtha and slower timed scores on trails $A$, and greater reports of negative affective symptoms on profile of mood states scales in $1988^{\circ}$ but not 1989. Threshold model analyses of the 1989 data showed an association between score on visual reproductions immediate recall and daily exposure to naphtha at or above $1050 \mathrm{~h} \times \mathrm{mg} / \mathrm{m}^{3}$. Models of chronic exposure showed no associations between chronic exposure and negative neurobehavioural outcome. Results suggest that naphtha produces mild acute reversible effects on function of the central nervous system at or above daily exposures of $540 \mathrm{~h} \times \mathrm{mg} / \mathrm{m}^{3}$ (approximately $90 \mathrm{ppm} / \mathrm{h}$ ).
\end{abstract}

(Occup Environ Med 1994;51:102-112)

Although there is still much to be learned about the specific variables associated with adverse health outcomes after exposure to solvents, ${ }^{1}$ there are several publications with different types of methodology that agree that solvents are neurotoxic. ${ }^{2-4}$ For example, animal studies have verified the neurotoxicity of trichloroethylene $e^{5-10}$ and benzene. ${ }^{11}$ Human studies with acute exposure have confirmed central nervous system and behavioural effects of exposure to solvents, ${ }^{12-14}$ as have case control studies of neuropsychiatric outcome, ${ }^{15-22}$ retrospective comparisons of test performance in solvent exposed workers and controls, ${ }^{23-28}$ and prospective assessments of the relations between exposure and neuropsychological outcome. ${ }^{189-40}$

Although naphtha, the solvent investigated in our study, has been described as a neurotoxicant and targeted as a solvent for neurobehavioural investigations, ${ }^{41}$ there has been little scientific examination of the behavioural effects of naphtha in humans. In one study, acute exposure to Stoddard solvent in normal non-occupationally exposed subjects showed that olfactory thresholds for the solvent were low but irritative effects were none the less minimal at exposures of one to four times the threshold limit value (TLV) of $100 \mathrm{ppm}^{42}$ These investigators found no dose effect relation between exposure and impaired performance on the Purdue pegboard (acute exposure to one TLV for 30 minutes), or the Michigan eye-hand coordination test, or choice reaction time (acute exposures of one to four times the TLV). An acute exposure study of a closely related solvent, white spirits, assessed effects of exposures ranging from $625-2500 \mathrm{mg} / \mathrm{m}^{3}$ over a 30 minute period. ${ }^{43}$ Although nine of the 14 subjects reported subjective symptoms of intoxication such as dizziness, none showed performance decrements on the behavioural tests used (perceptual speed, visual reaction time, memory, manual dexterity, digit addition). When eight subjects were exposed to $4000 \mathrm{mg} / \mathrm{m}^{3}$, however, short term memory and measures of simple reaction time were negatively affected. A case study describing health effects of exposure to mineral spirits in a worker with respiratory and dermal exposure (he placed his hands in solvent several times a day) reported symptoms of the central nervous system such as nervousness and headaches after three years of exposure, when he left employment because of ill health. ${ }^{44}$ One of us (RFW) has also seen a patient with clinical evidence of substantial damage to the central nervous system after more than 30 years of chronic occupational exposure to Stoddard solvent. Our review of publications on naphtha and related solvents suggested that naphtha is neurotoxic but substantial exposure may be required to produce acute or chronic effects.

Based on knowledge accumulated to date on the neuropsychological effects of exposure to solvents and our own research and clinical experience with subjects exposed to solvents, we have noted that exposure to solvents in adults particularly affects attention, executive function, visuospatial abilities, short term 
Table 1 Neuropsychological tests used

\begin{tabular}{|c|c|c|}
\hline Test & Functional domain & Cerebral localisation \\
\hline \multicolumn{3}{|c|}{1988} \\
\hline Trails A and B & $\begin{array}{l}\text { Attention (A) } \\
\text { Executive function (B) }\end{array}$ & Frontal system \\
\hline Profile of mood states & Mood states & Frontal, limbic \\
\hline Recognition span test & Short term memory & Limbic \\
\hline Visual reproduction & Short term memory & Limbic \\
\hline Vocabulary (NES)^ & General intelligence & (Hold test-none) \\
\hline Symbol-digit (NES)^ & $\begin{array}{l}\text { Motor, attention, } \\
\text { scanning, visuospatial }\end{array}$ & ?Motor strip \\
\hline Pattern memory (NES) ${ }^{\star}$ & Short term memory & ?Right hemisphere, limbic \\
\hline Wisconsin card sorting test & Executive & Frontal \\
\hline \multicolumn{3}{|c|}{1989} \\
\hline $\begin{array}{l}\text { As above except: } \\
\text { No vocabulatory (NES) } \\
\text { No Wisconsin card sorting test }\end{array}$ & & \\
\hline Rey-Osterreith complex figure & $\begin{array}{l}\text { Executive, visuospatial } \\
\text { short term memory }\end{array}$ & Frontal (planning), limbic \\
\hline
\end{tabular}

Tests from the neurobehavioural evaluation system (NES) have not yet been validated on patients with known localised brain damage; localisations are hypothesised based on studies with similar standard paper and pencil tests.
Table 2 Effects of solvents on the central nervous system. patterns of functional neuropsychological deficit

\begin{tabular}{lll}
\hline Effects & Acute & Chronic \\
\hline Mild: & Rev & Res \\
Mood & Rev & Res \\
Attention & Rev & Res \\
Executive function & & \\
Moderate: & P to F rev & - \\
$\quad$ Mood & P to F rev & - \\
Attention & P to F rev & - \\
Executive function & P to F rev & Res ME + \\
Visuospatial & P to F rev & Res ME + \\
Short term memory & P to F rev & Res ME + \\
Motor & & \\
Severe: & - & Res Mod E+ \\
Amnestic syndrome & - & \\
\hline
\end{tabular}

Rev = reversible; Res = residual; $P$ to $F$ rev = partially to fully reversible; Res $\mathrm{ME}+=$ residual, mild effects plus; Res Mod $\mathrm{E}+=$ residual moderate effects plus.

memory, and mood, ${ }^{45}$ sparing functions such as language and retrograde memory. These findings suggest that solvents affect the brain in a focal manner, not diffusely as has been previously proposed. Specifically, $\mathrm{we}^{346}$ and others $^{27}$ have theorised that solvents preferentially affect the frontal lobes and limbic system. In keeping with this prediction, the tests for our study were selected to include hold tests, that is - tests thought to be resistant to effects of solvents, as well as tasks specifically designed to assess frontal and limbic function (table 1). Also, our model of acute and chronic effects of exposure to solvents is based on prior work by one of us delineating the patterns of functional cognitive deficits expected in a model of acute and chronic effects of solvents (table 2). ${ }^{478}$ The current study was designed to provide as sensitive a group of measures as possible to determine whether there are neuropsychological deficits associated with exposure to naphtha and to allow assessment of a broad range of potential outcomes of exposure to naphtha on the central nervous system in a working population, ranging from mild reversible signs of dysfunction to moderate effects of chronic exposure.
Materials and methods

STUDY DESIGN

The study was a prospective assessment of neuropsychological function in workers with relatively high exposure to naphtha and a comparison group with low exposure at two times separated by a one year interval. Subjects were recruited from among the $\mathbf{5 3 3}$ hourly production workers on first and second shift. Over $90 \%$ of these workers were successfully contacted by in plant recruiters in an attempt to enlist 250 workers, 125 each from the area of relatively high exposure to naphtha (inside the three calibration rooms) and the area of relatively low exposure (outside the calibration rooms). In June 1988, 248 workers participated, 119 from inside the calibration rooms and 129 from outside. Reasons for declining to participate were not collected on a systematic basis. The sex and seniority data of all hourly production workers were obtained. Seniority date was the date of hire at the plant, or if the worker had been employed previously at another plant in the corporation, an earlier date determined by contact, usually within one year of date of hire at any company plant.

In June 1989,185 of the original 248 subjects participated again, 87 from inside the calibration rooms and 98 from outside. Among those lost to follow up, 33 refused outright, four were "busy", nine were on medical leave, seven were absent on two scheduled test dates, four were on vacation or training, and three had recently changed shift and could not be rescheduled.

\section{EXPOSURE TO NAPHTHA}

The blend of naphtha used at the plant consisted by weight of about $50 \%$ paraffins, $25 \%$ monocyclic naphthenes, $18 \%$ benzenes and $<5 \%$ each of dicyclic naphthenes, indans or teralins, naphthalenes, and olefins. Trimethylbenzene, methylethylbenzene, and diethylbenzene were the most prevalent constituents in the aromatic fraction.

Methods of characterisation of exposure and calculation of cumulative measures of exposure have been described elswhere. ${ }^{49}$ Briefly, cumulative exposure (years $\times \mathrm{mg} / \mathrm{m}^{3}$ ) was the product of the time worked in a naphtha exposure zone in a particular month and the mean naphtha air concentration in that zone that month, summed over the zones and months. Computerised work histories supplemented by personal interviews identified the dates worked in the zones. Mean naphtha air concentrations were calculated from 514 personal air samples for naphtha collected in June 1988, October 1988, December 1988, and June 1989. The sampling and analytical scheme was the National Institute of Occupational Safety and Health (NIOSH) method $1550 . .^{50}$ Due to a strong association between air concentration and outdoor temperature, mean concentrations for each month between February 1982 (plant opening) to June 1988 were adjusted for monthly changes in outdoor temperature. Recent exposure was calculated as the mean 
naphtha concentration of the exposure zone in which the employee worked currently adjusted for the mean temperature during that month.

Three alternative measures of the cumulative and recent exposure variables were calculated, based respectively on delineations of jobs at the plant into two, six, or 23 exposure zones. ${ }^{49}$ Results from the three types of measures were similar; only results from the scheme with six zones are presented here.

An investigation of the potential effect of skin absorption of naphtha on total hydrocarbon and trimethylbenzene concentrations in exhaled breath among this study population failed to show any significant or consistent effects (unpublished data). Therefore, calculated exposures to naphtha were not adjusted for potential dermal absorption.

Two measures of acute exposure to naphtha were examined: the simple TWA concentration and the product of the TWA times the number of hours worked on the day of testing before undergoing the tests. Because a greater number of significant associations were present with the product term and because toxicokinetic considerations suggested that this measure was more likely to reflect acute dose to the target organ central nervous system, only the product term results are presented here.

\section{IN PLANT EXPOSURE TO OTHER POTENTIAL}

\section{NEUROTOXICANTS}

As well as naphtha, subjects could be exposed to other potential neurotoxicants at the plant These substances included 1,1,2-trichloro1,2,2-trifluoroethane (Freon 113), low odour paraffin solvent, toluene, ethylene glycol monoethyl ether acetate, and cadmium. These exposures seemed unlikely to act as confounders because exposures were very low: in each case, $\leqslant 3.0 \%$ of the TLV designated by the American Conference of Governmental Industrial Hygienists (ACGIH) ${ }^{50}$ Of these Freon 113 was the most prevalent. Freon 113 concentrations among 13 workers expected to have the highest exposures ranged from 32 to $230 \mathrm{mg} / \mathrm{m}^{3}$ (ACGIH TLV is $7670 \mathrm{mg} / \mathrm{m}^{3}$ ). ${ }^{50}$ The sampling and analytical scheme used was NIOSH method $1020 . .^{51}$

\section{MEASURES OF BEHAVIOURAL FUNCTION}

During both data sampling periods, a questionnaire was given to each participant to assess medical history, including neurological and psychiatric disorders, renal disorders, hypertension, diabetes, use of medications, occupational history, educational history (including presence of learning problems), and history of alcohol consumption. The health questionnaire also included questions about the frequency and presence of transient and chronic symptoms associated with neurotoxic exposures. Pretesting and post-testing questionnaires were also given to assess subjective psychological and somatic symptoms experienced on the day of testing. Data were also collected from personnel records on the amount of overtime worked in the months and week before testing. The number of hours worked before the test was noted. After giving the questionnaire, the formal neuropsychological tests were completed. These comprised:

profile of mood states, (POMS) ${ }^{52}$-This is a self administered measure of mood consisting of six scales: tension, depression, anger, fatigue, confusion, and vigour.

trail making test, (trails) ${ }^{53}$-This is a test of timed sequencing that requires the subject to complete a simple sequence (A) and alternate sequencing in a spatial array (B). It is sensitive to solvents ${ }^{272854}$ and to frontal lobe functions. ${ }^{55}$

delayed recognition span test $t^{5}$ - This verbal and spatial learning test assesses both recognition and recall memory known to be sensitive to memory dysfunction. It requires pointing out new stimuli in an increasingly numerous array and incidental recall of verbal stimuli at 15 seconds and at two minutes.

visual reproductions - This is a subtest of the Wechsler memory scale, ${ }^{57}$ a task of visual memory that requires the subject to draw designs from immediate and delayed recall. It is sensitive to damage of the limbic system. ${ }^{58}$ symbol-digit substitution test-This is a subtest of the neurobehavioural evaluation system (NES) $)^{59}$ that assesses coding ability and motor speed. Its brain localisation is unknown at present but the task is sensitive to exposure to neurotoxicants. ${ }^{61}$

pattern memory,-This subtest of the NES uses visual stimulus arrays to assess visual memory. Its brain localisation is unknown but it is sensitive to exposure to neurotoxicants. ${ }^{14}$

vocabulary - This is an NES task designed to evaluate verbal knowledge and ability thought to be insensitive to neurotoxic exposures in adults. Because the subjects in this study objected to the task (they found it difficult and frustrating on the harder items), the Information subtest of the Wechsler adult intelligence scale revised (WAIS-R) ${ }^{62}$ was substituted during 1989 . This test also measures crystallised intelligence and is insensitive to moderate exposures to neurotoxicant in adults ${ }^{45}$ 63:

Wisconsin card sorting test (WCST) ${ }^{64}$-This test assesses concept formation, cognitive flexibility, and cognitive tracking and requires inferring decision rules for a sorting task with minimal examiner feedback. It is known to be sensitive to frontal lobe function. ${ }^{65}$ Because we believed that there would be a practice effect in repeating this task, it was only administered during 1988. In 1989, the following was used:

Rey-Osterreith complex figure task ${ }^{66}$ - This test requires drawing an intricate visual design to copy and from immediate and delayed recall. It measures constructional function, strategy development, and memory.

\section{POTENTIAL CONFOUNDERS}

The potential confounders considered in the regression models of outcomes on the central nervous system included age, sex, 
handedness, native language, highest grade completed in school, hours of overtime in the previous week or day, self reported use of psychotropic medications, frequency of marijuana use, history of alcohol abuse, history of loss of consciousness, history of seizures, history of emotional illness, familiarity with video display terminals, history of repeating a grade in school, and histories of prior exposure to lead, mercury, or solvents. Multiple regression was stepwise, missing values were deleted pairwise, and the $p$ value to enter was $0 \cdot 1$. The acute and cumulative measures of exposure were forced into the regression models as the final step.

The NES vocabulary scores were significantly negatively associated with cumulative exposure. Because vocabulary scores may be associated with several other neuropsychological scores and are expected to be highly resistant to solvent neurotoxic effects, key neuropsychological outcomes were reexamined in multiple regression models with vocabulary as a covariate.

\section{STATISTICAL ANALYSES}

Statistical analyses were conducted with SPSS-X version 3.0.67 Participants in 1988 were compared with non-participants, and second year participants were compared with those lost to follow up by means of $\chi^{2}$ tests (two tailed without Yates' correction) for dichotomous variables and the MannWhitney $U$ test for continuous variables. A paired $t$ test was used to examine differences in measures of neuropsychological function in 1988 and 1989.

For the cross sectional and longitudinal studies, the association between the measures of behavioural function and exposure to naphtha was examined with simple and multiple regression. Multiple regression was stepwise, the $\mathrm{p}$ value to enter was $0 \cdot 1$, and variables of exposure to naphtha were forced in as the final step. Independent variables included cumulative and recent exposure to naphtha and potential confounders. Multiple regression models including first order interaction terms between exposure to naphtha and potential confounders were also examined.

In view of the substantial differences found in regression models of neuropsychological outcomes in the two years of data collection and the substantially higher mean exposures in the first year, additional analyses were conducted. Firstly, the scores on all neuropsychological tests and symptom questions among the 185 subjects participating in both years were compared by paired $t$ test. To investigate threshold effects, the multiple regression models for the two sets of data were re-examined with the first year's acute exposure 75th percentile $\left(540 \mathrm{~h} \times \mathrm{mg} / \mathrm{m}^{3}\right)$, and separately the first year 90 percentile $\left(1050 \mathrm{~h} \times \mathrm{mg} / \mathrm{m}^{3}\right)$ as thresholds. For these models, the measure of acute exposure was recalculated as follows:

Acute exposure $(75$ th percentile threshold $)=$ 0 , if acute exposure $\leqslant 540 \mathrm{~h} \mathrm{x} \mathrm{mg/ \textrm {m } ^ { 3 }}$

Acute exposure (75th percentile threshold) $=$ acute exposure-540, if acute exposure $>540 \mathrm{~h} \times \mathrm{mg} / \mathrm{m}^{3}$

The acute exposure 90th percentile threshold was calculated in an analogous manner with a threshold of $1050 \mathrm{~h} \times \mathrm{mg} / \mathrm{m}^{3}$. These models test the hypothesis that acute exposure has no effect on outcomes at or below the threshold but has a positive linear effect on outcomes above the threshold. All standard and threshold regression models were examined for the influence of outliers.

Table 3 Comparison of mean (SD) on neuropsychological tests in 1988 and 1989

\begin{tabular}{|c|c|c|c|c|c|}
\hline Dependent variable & No & 1988 & 1989 & $\begin{array}{l}\text { p Value } \\
\text { (paired t test) }\end{array}$ & $\begin{array}{l}\text { Year of } \\
\text { poorer } \\
\text { performance }\end{array}$ \\
\hline $\begin{array}{l}\text { Trails A: } \\
\quad \text { Response time (s) } \\
\text { Errors (n) }\end{array}$ & $\begin{array}{l}185 \\
184\end{array}$ & $\begin{array}{l}30 \cdot 9(10 \cdot 4) \\
0.201(0.510)\end{array}$ & $\begin{array}{l}28 \cdot 5(9 \cdot 2) \\
0.114(0.381)\end{array}$ & $\begin{array}{l}0.002 \\
0.052\end{array}$ & $\begin{array}{l}1 \\
1\end{array}$ \\
\hline $\begin{array}{l}\text { Trails B: } \\
\quad \text { Response time (s) } \\
\text { Errors (n) }\end{array}$ & $\begin{array}{l}178 \\
176\end{array}$ & $\begin{array}{c}75 \cdot 7(39 \cdot 5) \\
0.78(1.68)\end{array}$ & $\begin{array}{c}74 \cdot 8(45 \cdot 8) \\
1.05(2 \cdot 80)\end{array}$ & $\begin{array}{l}0.81 \\
0.22\end{array}$ & \\
\hline $\begin{array}{l}\text { Moss: } \\
\text { Verbal scan } \\
\text { Verbal span total score } \\
15^{\prime \prime} \text { Verbal recall } \\
2^{\prime} \text { Verbal recall } \\
\text { Spatial scan } \\
\text { Spatial scan total score }\end{array}$ & $\begin{array}{l}184 \\
184 \\
184 \\
184 \\
184 \\
184\end{array}$ & $\begin{aligned} 12.91 & (2.45) \\
13.56 & (1.26) \\
5.87 & (1.58) \\
5.00 & (1.74) \\
11.46 & (2.56) \\
12.74 & (1.50)\end{aligned}$ & $\begin{aligned} & 13 \cdot 10(2 \cdot 18) \\
& 13 \cdot 75(0 \cdot 57) \\
& 7 \cdot 10(2 \cdot 25) \\
& 6 \cdot 13(2 \cdot 17) \\
& 12 \cdot 10(2 \cdot 22) \\
& 13 \cdot 16(1 \cdot 04)\end{aligned}$ & $\begin{array}{l}0.41 \\
0.053 \\
0.0005 \\
0.0005 \\
0.006 \\
0.001\end{array}$ & $\begin{array}{l}1 \\
1 \\
1 \\
1 \\
1\end{array}$ \\
\hline $\begin{array}{l}\text { Visual reproduction: } \\
\text { Immediate recall } \\
\text { Delayed recall }\end{array}$ & $\begin{array}{l}185 \\
185\end{array}$ & $\begin{array}{ll}8.79 & (2 \cdot 45) \\
7.80 & (2 \cdot 75)\end{array}$ & $\begin{array}{l}10.75(2.42) \\
10 \cdot 12(2.55)\end{array}$ & $\begin{array}{l}0.0005 \\
0.0005\end{array}$ & $\begin{array}{l}1 \\
1\end{array}$ \\
\hline $\begin{array}{l}\text { Profile of moods states: } \\
\text { Tension, } t \text { score } \\
\text { Depression, } t \text { score } \\
\text { Anger, } t \text { score } \\
\text { Fatigue, } t \text { score } \\
\text { Confusion, } t \text { score }\end{array}$ & $\begin{array}{l}184 \\
184 \\
184 \\
184 \\
184\end{array}$ & $\begin{array}{ll}38 \cdot 14 & (6 \cdot 85) \\
38 \cdot 47 & (4 \cdot 84) \\
43 \cdot 67 & (6 \cdot 45) \\
46 \cdot 41 & (7 \cdot 18) \\
38 \cdot 99 & (5 \cdot 22)\end{array}$ & $\begin{array}{ll}38 \cdot 70 & (6 \cdot 75) \\
38.41 & (4 \cdot 41) \\
44.07 & (6 \cdot 44) \\
45 \cdot 80 & (6.92) \\
39 \cdot 56 & (5 \cdot 34)\end{array}$ & $\begin{array}{l}0 \cdot 28 \\
0 \cdot 87 \\
0 \cdot 44 \\
0 \cdot 23 \\
0 \cdot 14\end{array}$ & \\
\hline $\begin{array}{l}\text { Pattern memory: } \\
\text { \% Correct } \\
\text { Response time (s) }\end{array}$ & $\begin{array}{l}184 \\
184\end{array}$ & $\begin{array}{c}80 \cdot 6(11 \cdot 9) \\
9 \cdot 11(2 \cdot 46)\end{array}$ & $\begin{array}{c}79 \cdot 9(11 \cdot 4) \\
8 \cdot 10(2 \cdot 23)\end{array}$ & $\begin{array}{l}0.51 \\
0.0005\end{array}$ & 1 \\
\hline $\begin{array}{l}\text { Symbol-digit: } \\
\text { \% Correct } \\
\text { Response time (s) }\end{array}$ & $\begin{array}{l}183 \\
183\end{array}$ & $\begin{array}{l}99 \cdot 2(2 \cdot 7) \\
23.61 \quad(6 \cdot 22)\end{array}$ & $\begin{array}{l}99 \cdot 0(3 \cdot 1) \\
22 \cdot 58(4 \cdot 82)\end{array}$ & $\begin{array}{l}0.68 \\
0.006\end{array}$ & 1 \\
\hline
\end{tabular}


Table 4 Comparison of mean (SD) symptom scores in 1988 and 1989

\begin{tabular}{|c|c|c|c|c|c|}
\hline Dependent variable & No & 1988 & 1989 & $\begin{array}{l}\text { p Value } \\
\text { (paired t test) }\end{array}$ & $\begin{array}{l}\text { Year of } \\
\text { poorer } \\
\text { performance }\end{array}$ \\
\hline Feeling tired & 180 & $2 \cdot 77^{\star}(0.86) \dagger$ & $2.53(0.87) \dagger$ & 0.0005 & 1 \\
\hline Difficulty concentrating & 180 & $1.81(0.76)$ & $1.86(0.70)$ & 0.36 & \\
\hline Having headaches & 180 & $1.93(0.94)$ & $1.93(0.88)$ & 0.93 & \\
\hline Difficulty falling asleep & 180 & $1.44(0.74)$ & $1.59(0.85)$ & 0.02 & 2 \\
\hline Diminished sexual desire & 179 & $1.38(0.70)$ & $1.41(0.67)$ & 0.51 & \\
\hline Tingling in fingers, toes & 179 & $1.44(0.80)$ & $1.38(0.70)$ & $0 \cdot 36$ & \\
\hline Facial numbness & 180 & $1.07(0.35)$ & $1.07(0.34)$ & 0.99 & \\
\hline Diminished appetite & 180 & $1.29(0.63)$ & $1.27(0.61)$ & $0 \cdot 72$ & \\
\hline Diarrhoea & 179 & $1.40(0.67)$ & $1.33(0.63)$ & $0 \cdot 13$ & \\
\hline Dry mouth & 182 & $1.66(0.88)$ & $1.52(0.71)$ & 0.03 & 1 \\
\hline Confusion & 180 & $1.23(0.52)$ & $1.35(0.63)$ & 0.007 & 2 \\
\hline Feeling depressed with or without reason & 181 & $1.50(0.75)$ & $1.40(0.63)$ & 0.08 & $\overline{1}$ \\
\hline Have to take notes to remember & 182 & $1.80(0.88)$ & $1.93(0.90)$ & 0.06 & 2 \\
\hline Hallucinations & 182 & $1.02(0.08)$ & $1.07(0.28)$ & 0.02 & 2 \\
\hline Heart palpitations & 179 & $1.30(0.70)$ & $1.29(0.63)$ & 0.89 & \\
\hline Experienced lack of coordination & 180 & $1.24(0.59)$ & $1.25(0.52)$ & $0 \cdot 88$ & \\
\hline Sleeping more than usual & 182 & $1.50(0.84)$ & $1.32(0.64)$ & 0.001 & 1 \\
\hline Skin dryness & 182 & $1.82(0.97)$ & $1.81(0.92)$ & 0.93 & \\
\hline Unexplained weight loss & 181 & $1.08(0.37)$ & $1.11(0.42)$ & 0.49 & \\
\hline Indigestion & 180 & $1.62(0.85)$ & $1.49(0.77)$ & 0.009 & 1 \\
\hline Excessive salivation & 178 & $1 \cdot 14(0.45)$ & $1.11(0.41)$ & 0.36 & \\
\hline Feeling irritable & 181 & $1.90(0.82)$ & $1.77(0.79)$ & 0.04 & 1 \\
\hline Light headedness & 181 & $1.40(0.69)$ & $1.31(0.59)$ & 0.05 & 1 \\
\hline Lack of muscle strength & 180 & $1.33(0.65)$ & $1.33(0.59)$ & 0.91 & \\
\hline Feeling excitable & 182 & $1.53(0.73)$ & $1.58(0.71)$ & $0 \cdot 38$ & \\
\hline Tightness in chest & 182 & $1.38(0.73)$ & $1.29(0.61)$ & 0.06 & 1 \\
\hline Nausea & 182 & $1.31(0.61)$ & $1.27(0.61)$ & $0 \cdot 15$ & \\
\hline Inflamed gums & 181 & $1.27(0.44)$ & $1 \cdot 10(0.34)$ & 0.54 & \\
\hline Tremor in fingers & 180 & $1.17(0.46)$ & $1.14(0.42)$ & 0.56 & \\
\hline Feeling anxious & 180 & $1.57(0.77)$ & $1.58(0.75)$ & 0.85 & \\
\hline Loose teeth & 179 & $1.03(0.25)$ & $1.04(0.19)$ & 0.53 & \\
\hline Trembling eyelids, lips, tongue & 181 & $1.22(0.52)$ & $1.19(0.51)$ & 0.64 & \\
\hline
\end{tabular}

Mean symptom score in past month: $1=$ not at all; $2=$ a little; $3=$ a moderate amount; $4=$ quite a lot. †SEM.

Table 5 Demographic variables and mean (SD) test scores stratified by acute naphtha exposure groups 1988

\begin{tabular}{|c|c|c|c|}
\hline \multirow[b]{2}{*}{ Dependent variable } & \multicolumn{3}{|c|}{ Acute naphtha exposure group } \\
\hline & $\begin{array}{l}75 \text { th } \\
\text { Percentile } \\
\left(<550 \mathrm{mg} / \mathrm{h} / \mathrm{m}^{3}\right) \\
(n=186)\end{array}$ & $\begin{array}{l}\text { 75th-90th } \\
\text { Percentile } \\
(n=36)\end{array}$ & $\begin{array}{l}>90 t h \\
\text { Percentile } \\
\left(>1060 \mathrm{mg} / \mathrm{h} / \mathrm{m}^{3}\right) \\
(n=25)\end{array}$ \\
\hline $\begin{array}{l}\% \text { Men } \\
\text { Age (y) } \\
\text { Education (y) } \\
\text { Overtime in week before testing (h) }\end{array}$ & $\begin{aligned} 64 \cdot 0 & \\
36 \cdot 0 & (0 \cdot 7)^{\star} \\
12 \cdot 4 & (0 \cdot 1) \\
9 \cdot 1 & (0 \cdot 6)\end{aligned}$ & $\begin{aligned} 61 \cdot 1 & \\
35 \cdot 1 & (1 \cdot 2)^{\star} \\
12 \cdot 5 & (0 \cdot 2) \\
5 \cdot 7 & (1 \cdot 2)\end{aligned}$ & $\begin{aligned} 60 \cdot 0 \\
36 \cdot 0(1 \cdot 8)^{\star} \\
12 \cdot 6(0 \cdot 3) \\
5 \cdot 1(0 \cdot 9)\end{aligned}$ \\
\hline $\begin{array}{l}\text { Trails A: } \\
\quad \text { Response time (s) } \\
\text { Errors (n) }\end{array}$ & $\begin{array}{l}31.1(0.9) \\
0.20(0.04)\end{array}$ & $\begin{array}{l}29.3(1.2) \\
0.14(0.06)\end{array}$ & $\begin{array}{l}37 \cdot 8(4 \cdot 4) \\
0.12(0.07)\end{array}$ \\
\hline $\begin{array}{l}\text { Trails B: } \\
\quad \text { Response time (s) } \\
\text { Errors (n) }\end{array}$ & $\begin{array}{l}79.8(3.6) \\
0.85(0.14)\end{array}$ & $\begin{array}{l}78 \cdot 1(5 \cdot 5) \\
1.12(0.42)\end{array}$ & $\begin{array}{l}76.5(4.9) \\
0.58(0.27)\end{array}$ \\
\hline $\begin{array}{l}\text { Wisconsin card sorting test: } \\
\text { Correct sets (n) } \\
\text { Errors (n) } \\
\text { Times sets lost (n) }\end{array}$ & $\begin{array}{l}3 \cdot 16(0 \cdot 10) \\
13 \cdot 3(0 \cdot 8) \\
2 \cdot 04(0 \cdot 14)\end{array}$ & $\begin{array}{l}3 \cdot 36(0 \cdot 20) \\
12 \cdot 1(1 \cdot 5) \\
1 \cdot 78(0 \cdot 29)\end{array}$ & $\begin{array}{c}3.59(0.23) \\
11.0(1 \cdot 8) \\
1.73(0.30)\end{array}$ \\
\hline $\begin{array}{l}\text { Moss: } \\
\text { Verbal span } \\
\text { Verbal span total score } \\
15^{\prime \prime} \text { Verbal recall } \\
2^{\prime} \text { Verbal recall } \\
\text { Spatial span } \\
\text { Spatial span total score }\end{array}$ & $\begin{array}{l}12 \cdot 9(0 \cdot 2) \\
13 \cdot 6(0 \cdot 1) \\
5 \cdot 86(0 \cdot 12) \\
4 \cdot 93(0 \cdot 14) \\
11 \cdot 3(0 \cdot 2) \\
12 \cdot 8(0 \cdot 1)\end{array}$ & $\begin{array}{l}13 \cdot 7(0 \cdot 2) \\
13 \cdot 8(0 \cdot 1) \\
5 \cdot 94(0 \cdot 28) \\
5 \cdot 31(0 \cdot 25) \\
11 \cdot 3(0 \cdot 4) \\
12 \cdot 7(0 \cdot 2)\end{array}$ & $\begin{array}{l}12 \cdot 7(0.5) \\
13.6(0.2) \\
6.79(0.46) \\
5 \cdot 75(0.46) \\
11.8(0.5) \\
13.0(0.3)\end{array}$ \\
\hline $\begin{array}{l}\text { Visual reproduction: } \\
\text { Immediate recall } \\
\text { Delayed recall }\end{array}$ & $\begin{array}{l}8.67(0.19) \\
7.75(0.20)\end{array}$ & $\begin{array}{ll}7.89 & (0.46) \\
6.83 & (0.53)\end{array}$ & $\begin{array}{l}8.88(0.49) \\
7.48 \quad(0.53)\end{array}$ \\
\hline $\begin{array}{l}\text { POMS: } \\
\text { Tension, } \mathrm{t} \text { score } \\
\text { Depression, } \mathrm{t} \text { score } \\
\text { Anger, } \mathrm{t} \text { score } \\
\text { Fatigue, t score } \\
\text { Confusion, t score }\end{array}$ & $\begin{array}{l}37 \cdot 8(0 \cdot 5) \\
38 \cdot 3(0 \cdot 3) \\
43.5(0.5) \\
45 \cdot 7(0.5) \\
38 \cdot 5(0.3)\end{array}$ & $\begin{array}{l}38 \cdot 3(1 \cdot 3) \\
38 \cdot 5(0 \cdot 9) \\
43 \cdot 8(1 \cdot 3) \\
47 \cdot 0(1 \cdot 4) \\
38 \cdot 9(1 \cdot 0)\end{array}$ & $\begin{array}{l}42 \cdot 1(1 \cdot 7) \\
40 \cdot 4(1 \cdot 1) \\
45 \cdot 4(1 \cdot 7) \\
47 \cdot 7(1 \cdot 9) \\
42 \cdot 8(1 \cdot 5)\end{array}$ \\
\hline $\begin{array}{l}\text { Pattern memory: } \\
\text { Percentage correct } \\
\text { Response time (s) }\end{array}$ & $\begin{array}{l}79.9(1.0) \\
9.02(0.18)\end{array}$ & $\begin{array}{l}77.8(1.8) \\
9.00(0.40)\end{array}$ & $\begin{array}{l}78.6(1.8) \\
9.18(0.49)\end{array}$ \\
\hline $\begin{array}{l}\text { Vocabulary } \\
\text { Symbol-digit: \% Correct } \\
\text { Response time (s) }\end{array}$ & $\begin{array}{l}71.0(1 \cdot 1) \\
99.0(0 \cdot 2) \\
23.7(0 \cdot 5)\end{array}$ & $\begin{array}{l}74 \cdot 3(2 \cdot 5) \\
99 \cdot 1(0 \cdot 6) \\
22 \cdot 5(0.6)\end{array}$ & $\begin{array}{l}72 \cdot 2(3 \cdot 1) \\
99 \cdot 3(0 \cdot 3) \\
25 \cdot 5(1 \cdot 5)\end{array}$ \\
\hline
\end{tabular}

\section{Results}

Air concentrations of naphtha ranged from 9 to $590 \mathrm{mg} / \mathrm{m}^{3}$ in June 1988 and 4 to 790 $\mathrm{mg} / \mathrm{m}^{3}$ in June 1989. Mean (SD) naphtha concentrations were significantly higher inside the calibration rooms than outside the calibration rooms and higher in June 1988 than in June 1989-namely, in June 1988 $177(71) \mathrm{mg} / \mathrm{m}^{3}$ inside and $40(39) \mathrm{mg} / \mathrm{m}^{3}$ outside and in June $1989127(55) \mathrm{mg} / \mathrm{m}^{3}$ inside and $31(14) \mathrm{mg} / \mathrm{m}^{3}$ outside. (As naphtha is a complex mixture of compounds of varying molecular weight, the relation of concentration in $\mathrm{mg} / \mathrm{m}^{3}$ to parts per million (ppm) may vary from one naphtha sample to another; however, division of $\mathrm{mg} / \mathrm{m}^{3}$ by six to obtain $\mathrm{ppm}$ is a reasonable approximation.) The calculated cumulative exposure to naphtha among participants averaged 216 (range $18-834) \times \mathrm{mg} / \mathrm{m}^{3}$ in June 1988 and 269 (range $37-905) \times \mathrm{mg} / \mathrm{m}^{3}$ in June 1989 . Duration of exposure averaged $2 \cdot 8$ (range $0 \cdot 8-6 \cdot 3$ ) years in June 1988 and 3.9 (range $1 \cdot 8-7 \cdot 3$ ) years in June 1989.

Compared with eligible non-participants, participants in 1988 had lower mean seniority (5.04 $v 6.53$ years, $\mathrm{p}<0.0005)$ but did not differ significantly with regard to sex $(66 \%$ men $v 57 \%$ women). Those lost to follow up in 1989 did not differ from those who participated in 1988 on potential confounding variables or exposure to naphtha except that second year participants were somewhat older in $1988(37 \cdot 1 v 34.7$ years, $\mathrm{p}=0.08)$ and had more exposure to organic solvents in jobs held before working at the plant $(3.8 v 0.1$ years, $\mathrm{p}<0.0005$ ).

COMPARISON OF SCORES IN 1988 AND 1989

Tables 3 and 4 compare the mean test and symptom scores for the two years. The mean 
Table 6 Mean scores for selected symptoms stratified by acute naphtha exposure groups 1988

\begin{tabular}{|c|c|c|c|}
\hline \multirow[b]{2}{*}{ Symptom } & \multicolumn{3}{|c|}{ Acute naphtha exposure group } \\
\hline & $\begin{array}{l}<75 \text { th } \\
\text { Percentile } \\
\left(<550 \mathrm{~h} / \mathrm{mg} / \mathrm{m}^{3}\right) \\
(n=186)\end{array}$ & $\begin{array}{l}\text { 75th-90th } \\
\text { Percentile } \\
(n=36)\end{array}$ & $\begin{array}{l}>90 \mathrm{th} \\
\text { Percentile } \\
\left(>1060 \mathrm{~h} / \mathrm{mg} / \mathrm{m}^{3}\right) \\
(n=25)\end{array}$ \\
\hline $\begin{array}{l}\text { Feeling tired } \\
\text { Difficulty concentrating } \\
\text { Having headaches } \\
\text { Dry mouth } \\
\text { Confusion } \\
\text { Feeling depressed without reason } \\
\text { Lack of coordination } \\
\text { Sleeping more than usual } \\
\text { Feeling irritable } \\
\text { Light headedness } \\
\text { Lack of muscle strength } \\
\text { Tightness in chest }\end{array}$ & $\begin{array}{l}2.79 \star(0.07) \dagger \\
1.80(0.05) \\
1.92(0.07) \\
1.55(0.06) \\
1.19(0.03) \\
1.50(0.05) \\
1.20(0.04) \\
1.42(0.06) \\
1.87(0.06) \\
1.40(0.05) \\
1.30(0.04) \\
1.28(0.05)\end{array}$ & $\begin{array}{l}2.28(0.14) \dagger \\
1.83(0.15) \\
2.19(0.19) \\
1.81(0.17) \\
1.22(0.10) \\
1.42(0.12) \\
1.42(0.15) \\
1.44(0.14) \\
1.72(0.15) \\
1.39(0.14) \\
1.39(0.12) \\
1.53(0.14)\end{array}$ & $\begin{array}{l}2.64(0.20) \\
2.00(0.15) \\
2.17(0.25) \\
2.00(0.22) \\
1.46(0.18) \\
1.96(0.20) \\
1.38(0.16) \\
1.75(0.22) \\
2.17(1.01) \\
1.54(0.18) \\
1.67(0.21) \\
1.54(0.18)\end{array}$ \\
\hline
\end{tabular}

*Mean symptom score in past month: $1=$ not at all; $2=$ a little; $3=$ a moderate amount; $4=$ quite a lot.

tSEM

test scores on the neuropsychological measures are generally within expected norms for a subject group with a mean age of 36 and a mean educational level of 12 years. Among the tests, in all cases of significant difference, the poorer performance was in 1988. Tests showing significant differences included trails A (response time and number of errors), most of the Moss verbal and spatial scores, visual reproductions (immediate and delayed recall), pattern memory response time, and

Table 7 Cross sectional multiple regression* analysis of neuropsychological outcomes for 1988

\begin{tabular}{|c|c|c|c|c|c|c|}
\hline \multirow[b]{2}{*}{ Dependent variable } & \multicolumn{3}{|c|}{ Acute exposure } & \multicolumn{3}{|c|}{ Cumulative exposure } \\
\hline & Coeff & SEM & p Value & Coeff & SEM & p Value \\
\hline $\begin{array}{l}\text { Trails A: } \\
\quad \text { Response time (s) } \\
\text { Errors (n) }\end{array}$ & 0.005 & 0.002 & $\begin{array}{l}0.024 \\
0.27\end{array}$ & -0.009 & 0.006 & $\begin{array}{l}0.12 \dagger \\
0.95\end{array}$ \\
\hline $\begin{array}{l}\text { Trails B: } \\
\quad \text { Response time (s) } \\
\text { Errors (n) }\end{array}$ & & & $\begin{array}{l}0.98 \\
0.93\end{array}$ & & & $\begin{array}{l}0.85 \\
0.58\end{array}$ \\
\hline $\begin{array}{l}\text { Wisconsin card sorting test } \\
\text { Correct sets (n) } \\
\text { Errors (n) } \\
\text { Times sets lost (n) }\end{array}$ & & & $\begin{array}{l}0 \cdot 75 \\
0 \cdot 76 \\
0 \cdot 25\end{array}$ & -0.008 & 0.005 & $\begin{array}{l}0 \cdot 36 \\
0 \cdot 10 \dagger \\
0 \cdot 86\end{array}$ \\
\hline $\begin{array}{l}\text { Moss: } \\
\text { Verbal span } \\
\text { Verbal span total score } \\
\text { 15" Verbal recall } \\
2^{\prime} \text { Verbal recall } \\
\text { Spatial span } \\
\text { Spatial span total score }\end{array}$ & 0.0004 & 0.0003 & $\begin{array}{l}0 \cdot 36 \\
0 \cdot 90 \\
0 \cdot 11 \dagger \\
0 \cdot 36 \\
0 \cdot 32 \\
0 \cdot 68\end{array}$ & & & $\begin{array}{l}0.55 \\
0.43 \\
0.96 \\
0.36 \\
0.63 \\
0.59\end{array}$ \\
\hline $\begin{array}{l}\text { Visual reproduction: } \\
\text { Immediate recall } \\
\text { Delayed recall }\end{array}$ & & & $\begin{array}{l}0.63 \\
0.36\end{array}$ & $\begin{array}{l}0.0018 \\
0.0021\end{array}$ & $\begin{array}{l}0.0012 \\
0.0012\end{array}$ & $\begin{array}{l}0.13 \dagger \\
0.09 \dagger\end{array}$ \\
\hline $\begin{array}{l}\text { POMS: } \\
\text { Tension, t score } \\
\text { Depression, t score } \\
\text { Anger, t score } \\
\text { Fatigue, t score } \\
\text { Confusion, t score }\end{array}$ & $\begin{array}{l}0.0022 \\
0.0016 \\
0.0020 \\
0.0031\end{array}$ & $\begin{array}{l}0.0011 \\
0.0008 \\
0.0010 \\
0.0008\end{array}$ & $\begin{array}{l}0.042 \\
0 \cdot 043 \\
0 \cdot 055 \\
0 \cdot 16 \\
0.0004\end{array}$ & & & $\begin{array}{l}0 \cdot 77 \\
0 \cdot 31 \\
0 \cdot 38 \\
0 \cdot 35 \\
0 \cdot 22\end{array}$ \\
\hline $\begin{array}{l}\text { Pattern memory: } \\
\% \text { Correct } \\
\text { Response time (s) }\end{array}$ & & & $\begin{array}{l}0.38 \\
0.62\end{array}$ & & & $\begin{array}{l}0.37 \\
0.41\end{array}$ \\
\hline $\begin{array}{l}\text { Vocabulary } \\
\text { Symbol-digit: } \\
\% \text { Correct } \\
\text { Response time (s) }\end{array}$ & & & $\begin{array}{l}0.52 \\
0.41\end{array}$ & 0.014 & 0.006 & $\begin{array}{l}0.02 \dagger \\
0.48 \\
0.98\end{array}$ \\
\hline
\end{tabular}

^Each line in the table represents a single regression model. Acute and cumulative exposure measures were included in each model. Covariates included if $p$ value $<0 \cdot 1$. Covariates considered included overtime worked in past week, age, sex, handedness, self report of effort, sleep in past 24 hours, caffeine in past 24 hours, alcohol in past 24 hours, use of marijuana, use of alcohol, history of loss of consciousness, history of seizures, history of emotional problems familiarity with video games, first language, education, repeated grades in school, history of mercury exposure, history of lead exposure, and history of solvent exposure. The only covariate shown is overtime worked in past week.

tEffect in counterintuitive direction. symbol digit response time. The findings for symptoms (table 4) were mixed. Among the 12 symptoms for which there were significant differences, eight were more frequent in 1988 and four in 1989.

COMPARISON OF DEMOGRAPHIC VARIABLES AND MEAN TEST AND SYMPTOM SCORES ACROSS ACUTE NAPHTHA EXPOSURE GROUPS

Table 5 shows that participants with acute exposure to naphtha less than the 75 th percentile in 1988 were similar to higher exposed participants with respect to age, sex, and years of education but had worked more overtime in the week before testing. Among the tests examined, trails $A$ response time and five POMS scores showed the most consistent trends of poorer performance with increasing exposure. Timed performance was somewhat slowed on trails $\mathrm{A}$ in the highest exposed group (average time for 20 to 39 year olds is 32 seconds $^{65}$ ). The Moss DRST 15 second verbal recall showed the strongest trend in a counter intuitive direction (improved performance with increasing exposure). Similar analyses of the 1989 data showed fewer apparent trends in test scores (not shown). Only for visual reproductions immediate recall and for symbol digit response times were there trends in the expected direction.

Table 6 shows that for subjective symptoms fatigue was the most intensely endorsed at all threshold levels. There were trends suggesting increasing frequencies of several symptoms with increasing exposure in 1988, particularly if the highest exposure group was compared with the other two groups. Trends were particularly notable for dry mouth, confusion, feeling depressed without reason, sleeping more than usual, and lack of muscle strength. Substantially fewer such trends were present for symptoms reported in 1989 (not shown).

\section{CROSS SECTIONAL MULTIPLE REGRESSION} ANALYSES

Neuropsychological test scores

Table 7 presents the results of the cross sectional multiple linear regression models of the neuropsychological outcomes in 1988. Trails A (number of errors and response time) and $t$ scores on four of the POMS affective scales (depression, anger, tension, and confusion) were significantly associated with the measure of acute exposure in 1988, although the significant association for trails A errors was due to an outlier. The Moss (Delayed Recognition Span Test) 15 second verbal recall was marginally associated with acute exposure in a counter intuitive direction. In 1989 (not shown), only the symbol digit subtest was significantly associated with the measure of acute exposure (coefficient 0.0026 $(0.0011) ; \mathrm{p}=0.014)$.

None of the tests was associated with the measure of cumulative exposure in the expected direction in either year. In 1988, trails A (response time and number of errors), Wisconsin card sort (number of errors) and 
Table 8 Cross sectional multiple regression* analysis of neuropsychological outcomes for 1988

\begin{tabular}{|c|c|c|c|c|c|c|}
\hline \multirow[b]{2}{*}{ Dependent variable } & \multicolumn{3}{|c|}{ Acute exposure } & \multicolumn{3}{|c|}{ Cumulative exposure } \\
\hline & Coeff & $S E M$ & p Value & Coeff & SEM & p Value \\
\hline Feeling tired & & & $0 \cdot 40$ & & & 0.38 \\
\hline Difficulty concentrating & & & 0.56 & & & $0 \cdot 16$ \\
\hline Having headaches & 0.00023 & 0.00015 & $0 \cdot 12$ & & & 0.32 \\
\hline $\begin{array}{l}\text { Difficulty falling asleep } \\
\text { Diminished sexual desire }\end{array}$ & & & $\begin{array}{l}0.45 \\
0.86\end{array}$ & & & $\begin{array}{l}0.50 \\
0.32\end{array}$ \\
\hline Tingling in fingers, toes & & & 0.99 & & & 0.78 \\
\hline Facial numbness & 0.00014 & 0.00006 & 0.01 & & & 0.83 \\
\hline Diminished appetite & & & 0.49 & & & 0.59 \\
\hline Diarrhoea & & & $0 \cdot 16$ & & & 0.96 \\
\hline Dry mouth & 0.00041 & 0.00013 & 0.002 & & & 0.95 \\
\hline Confusion & 0.00041 & 0.00008 & 0.09 & & & 0.94 \\
\hline $\begin{array}{c}\text { Feeling depressed } \\
\text { without reason }\end{array}$ & 0.00018 & 0.00012 & 0.15 & & & 0.48 \\
\hline Have to make notes & & & 0.13 & & & 0.48 \\
\hline to remember & & & 0.91 & & & 0.83 \\
\hline Hallucinations & & & 0.82 & & & 0.88 \\
\hline Heart palpitations & & & $0 \cdot 16$ & & & 0.57 \\
\hline Experienced lack of & & & & & & \\
\hline coordination & & & 0.33 & 0.00040 & 0.00026 & 0.13 \\
\hline Sleeping more than usual & 0.00019 & 0.00013 & $0 \cdot 12$ & & & 0.99 \\
\hline Skin dryness & 0.00025 & $0 \cdot 00016$ & $0 \cdot 11$ & 0.00064 & 0.00043 & $0 \cdot 15$ \\
\hline Unexplained weight loss & & & $0 \cdot 60$ & & & 0.62 \\
\hline Indigestion & 0.00024 & 0.00014 & $0 \cdot 14$ & & & 0.35 \\
\hline Excessive salivation & & & 0.96 & & & $0 \cdot 36$ \\
\hline Feeling irritable & & & $0 \cdot 84$ & & & $0 \cdot 37$ \\
\hline Light headedness & & & 0.44 & & & 0.52 \\
\hline Lack of muscle strength & 0.00021 & 0.00011 & 0.05 & & & 0.22 \\
\hline Feeling excitable & & & 0.72 & & & 0.91 \\
\hline Tightmess in chest & 0.00026 & 0.00011 & 0.02 & & & 0.63 \\
\hline Nausea & & & 0.78 & 0.00041 & 0.00028 & 0.015 \\
\hline Inflamed gums & & & 0.37 & 0.00043 & 0.00020 & 0.04 \\
\hline Tremor in fingers & & & 0.92 & & & 0.77 \\
\hline Feeling anxious & & & $0 \cdot 27$ & & & 0.44 \\
\hline Loose teeth & & & 0.90 & & & 0.35 \\
\hline Trembling eyelids, & & & & & & \\
\hline lips, tongue & 0.00022 & 0.00008 & 0.009 & & & 0.98 \\
\hline
\end{tabular}

^As for table 7 . visual reproductions (immediate and delayed recall) were marginally associated with estimated cumulative exposure in the counter intuitive direction. Vocabulary scores were significantly negatively associated with exposure (those who had the highest exposure had the better vocabulary scores). Because vocabulary scores may be associated with other scores and are expected to be highly resistant to neurotoxic effects, key outcomes were reexamined in multiple regression models including vocabulary as a covariate. In no case, however, did this adjustment have a meaningful effect on the association between the measure of exposure and outcomes.

\section{Symptoms}

Table 8 presents the results of cross sectional multiple linear regression analyses of subjective symptomatic complaints in 1988. Self reported symptoms showing significant associations with the measure of acute exposure during 1988 included facial numbness, dry mouth, lack of muscle strength, tightness in chest, facial trembling, and, marginally, confusion. Only a few subjective symptoms were associated with the measure of acute exposure in 1989-namely, diarrhoea, headache, and, marginally, difficulty concentrating (data not shown).

Table 91988 Cross sectional multiple regression * models of association of acute exposure measurest with neuropsychological outcomes

\begin{tabular}{|c|c|c|c|c|c|c|c|c|c|c|c|c|}
\hline \multirow[b]{2}{*}{ Dependent Variable } & \multicolumn{4}{|c|}{ No threshold $\ddagger$} & \multicolumn{4}{|c|}{ Threshold at 75 th percentile $\$$} & \multicolumn{4}{|c|}{ Threshold at 90th percentile } \\
\hline & Coeff & SEM & $p$ Value & Model $r^{2}$ & $\overline{C o e f f}$ & $S E M$ & $p$ Value & Model $r^{2}$ & Coeff & $S E$ & $p$ Value & Model $r^{2}$ \\
\hline $\begin{array}{l}\text { Trails A: } \\
\quad \text { Response time (s) } \\
\text { Errors (n) }\end{array}$ & 0.0050 & 0.0020 & $\begin{array}{l}0.022 \\
0.27\end{array}$ & $0 \cdot 154$ & 0.0101 & 0.0031 & $\begin{array}{l}0.0014 \\
0.65\end{array}$ & 0.173 & 0.0314 & 0.0070 & $\begin{array}{c}<0.0001 \\
0.95\end{array}$ & $0 \cdot 207$ \\
\hline $\begin{array}{l}\text { Trails B: } \\
\quad \text { Response time (s) } \\
\text { Errors (n) }\end{array}$ & & & $\begin{array}{l}0.97 \\
0.89\end{array}$ & & & & $\begin{array}{l}0.99 \\
0.70\end{array}$ & & & & $\begin{array}{l}0.88 \\
0.91\end{array}$ & \\
\hline $\begin{array}{l}\text { Wisconsin card sorting test: } \\
\text { Correct sets (n) } \\
\text { Errors (n) } \\
\text { Times sets lost (n) }\end{array}$ & & & $\begin{array}{l}0.75 \\
0.76 \\
0.25\end{array}$ & & & & $\begin{array}{l}0 \cdot 38 \\
0 \cdot 84 \\
0 \cdot 42\end{array}$ & & & & $\begin{array}{l}0.60 \\
0.84 \\
0.34\end{array}$ & \\
\hline $\begin{array}{l}\text { Moss: } \\
\text { Verbal span } \\
\text { Verbal span total score } \\
\text { 15" Verbal recall } \\
2^{\prime} \text { Verbal recall } \\
\text { Spatial span } \\
\text { Spatial span total score }\end{array}$ & 0.0004 & 0.0003 & $\begin{array}{l}0.36 \\
0.90 \\
0.109^{\star \star} \\
0.40 \\
0.32 \\
0.68\end{array}$ & $0 \cdot 118$ & 0.0007 & 0.0004 & $\begin{array}{l}0.64 \\
0.55 \\
0.080^{\star \star} \\
0.20 \\
0.50 \\
0.64\end{array}$ & 0.12 & 0.0011 & 0.0010 & $\begin{array}{l}0.78 \\
0.46 \\
0.26 \\
0.29 \\
0.98 \\
0.40\end{array}$ & 0.125 \\
\hline $\begin{array}{l}\text { Visual reproduction: } \\
\text { Immediate recall } \\
\text { Delayed recall }\end{array}$ & & & $\begin{array}{l}0.61 \\
0.44\end{array}$ & & & & $\begin{array}{l}0.85 \\
0.45\end{array}$ & & & & $\begin{array}{l}0.63 \\
0.70\end{array}$ & \\
\hline $\begin{array}{l}\text { POMS: } \\
\text { Tension, } t \text { score } \\
\text { Depression, } t \text { score } \\
\text { Anger, } t \text { score } \\
\text { Fatigue, } t \text { score } \\
\text { Confusion, } t \text { score }\end{array}$ & $\begin{array}{l}0.0022 \\
0.0016 \\
0.0020 \\
0.0027\end{array}$ & $\begin{array}{l}0.0011 \\
0.0008 \\
0.0010 \\
0.0008\end{array}$ & $\begin{array}{l}0.040 \\
0.043 \\
0.055 \\
0 \cdot 15 \\
0.002\end{array}$ & $\begin{array}{l}0 \cdot 137 \\
0 \cdot 15 \\
0 \cdot 166 \\
\\
0 \cdot 132\end{array}$ & $\begin{array}{l}0.0040 \\
0.0028 \\
0.0033 \\
0.0053\end{array}$ & $\begin{array}{l}0.0017 \\
0.0012 \\
0.0016 \\
0.0013\end{array}$ & $\begin{array}{l}0.018 \\
0.019 \\
0.039 \\
0.18 \\
0.0001\end{array}$ & $\begin{array}{l}0 \cdot 143 \\
0 \cdot 156 \\
0 \cdot 169 \\
0.159+t\end{array}$ & $\begin{array}{l}0.0078 \\
0.0046 \\
0.0079 \\
0.0110\end{array}$ & $\begin{array}{l}0.0038 \\
0.0029 \\
0.0036 \\
0.0029\end{array}$ & $\begin{array}{l}0.041 \\
0 \cdot 087 \\
0.030 \\
0.32 \\
0.0002\end{array}$ & $\begin{array}{l}0.137 \\
0.146 \\
0 \cdot 170 \\
0 \cdot 146\end{array}$ \\
\hline $\begin{array}{l}\text { Pattern memory: } \\
\text { \% Correct } \\
\text { Response time (s) }\end{array}$ & & & $\begin{array}{l}0.38 \\
0.62\end{array}$ & & & & $\begin{array}{l}0.25 \\
0.99\end{array}$ & & & & $\begin{array}{l}0.32 \\
0.52\end{array}$ & \\
\hline $\begin{array}{l}\text { Symbol-digit: } \\
\text { \% Correct } \\
\text { Response time (s) }\end{array}$ & 0.0006 & 0.0009 & $\begin{array}{l}0.52 \\
0.49\end{array}$ & 0.257 & 0.0020 & 0.0014 & $\begin{array}{l}0.60 \\
0.16\end{array}$ & 0.262 & 0.0068 & 0.0033 & $\begin{array}{l}0.94 \\
0.037\end{array}$ & $0 \cdot 270$ \\
\hline
\end{tabular}

*Each line in the table represents three separate regression models. Acute and cumulative exposure measures were included in each model. Covariates included if p value $<0 \cdot 1$. Covariates considered included overtime worked in past week, age, sex, handedness, self report of effort, sleep in past 24 hours, caffeine in past 24 hours, alcohol in past 24 hours, marijuana use, alcohol use, history of loss of consciousness, history of seizures, history of emotional problems, familiarity with video games, first language, education, repeated grades in school, history of mercury exposure, history of lead exposure, history of solvent exposure, and vocabulary. Only the results for the acute exposure measure are shown.

tAcute exposure $=$ measured concentration of naphtha on day of testing $\left(\mathrm{mg} / \mathrm{m}^{3}\right) \times$ on job before testing.

$\neq$ Acute exposure treated as a standard continuous variable.

Acute exposure $=0$, if $\leqslant 540 \mathrm{~h} \times \mathrm{mg} / \mathrm{m}^{3}(\mathrm{n}=188)$. Acute exposure $=$ measured value -540 , if $>540 \mathrm{~h} \times \mathrm{mg} / \mathrm{m}^{3}(\mathrm{n}=61)$

qAcute exposure $=0$, if $\leqslant 1050 \mathrm{~h} \times \mathrm{mg} / \mathrm{m}^{3}(\mathrm{n}=222)$. Acute exposure $=$ measured value -1050 , if $>1050 \mathrm{~h} \times \mathrm{mg} / \mathrm{m}^{3}(\mathrm{n}=25)$.

$\star \star$ Effect in counterintuitive direction.

t+Model $r^{2}$ not directly comparable to $r^{2}$ for no threshold because different set of covariates entered model. 
Table 10 Longitudinal multiple regression analysis of association of change in neurobehavioral outcomes with change in acute and cumulative exposures between 1988 and 1989

\begin{tabular}{|c|c|c|c|c|c|c|c|c|c|}
\hline \multirow[b]{2}{*}{ Dependent variable: (difference in) } & \multicolumn{3}{|c|}{ Difference in acute exposure } & \multicolumn{3}{|c|}{ Difference in cumulative exposure } & \multicolumn{3}{|c|}{ Cumulative exposure up to 1988} \\
\hline & Coeff & $S E$ & p Value & $\overline{C o e f f}$ & $S E$ & $p$ Value & $\overline{C o e f f}$ & $S E$ & p Value \\
\hline $\begin{array}{l}\text { Trail A: } \\
\quad \text { Response time(s) } \\
\text { Errors(n) }\end{array}$ & 0.0036 & 0.0023 & $\begin{array}{l}0.11 \\
0.27\end{array}$ & & & $\begin{array}{l}0.43 \\
0.76\end{array}$ & & & $\begin{array}{l}0.83 \\
0.54\end{array}$ \\
\hline $\begin{array}{l}\text { Trails B: } \\
\quad \text { Response time(s) } \\
\text { Errors(n) }\end{array}$ & & & $\begin{array}{l}0.66 \\
0.66\end{array}$ & & & $\begin{array}{l}0.36 \\
0.73\end{array}$ & & & $\begin{array}{l}0.95 \\
0.44\end{array}$ \\
\hline $\begin{array}{l}\text { Moss: } \\
\text { Verbal span } \\
\text { Verbal span total score } \\
\text { 15" Verbal recall } \\
2^{\prime} \text { Verbal recall } \\
\text { Spatial span } \\
\text { Spatial span total score }\end{array}$ & & & $\begin{array}{l}0.41 \\
0.35 \\
0.25 \\
0.50 \\
0.20 \\
0.21\end{array}$ & 0.014 & 0.08 & $\begin{array}{l}0.065 \\
0.42 \\
0.48 \\
0.98 \\
0.31 \\
0.87\end{array}$ & & & $\begin{array}{l}0.72 \\
0.87 \\
0 \cdot 19 \\
0 \cdot 20 \\
0.30 \\
0.47\end{array}$ \\
\hline $\begin{array}{l}\text { Visual reproduction: } \\
\text { Immediate recall } \\
\text { Delayed recall }\end{array}$ & & & $\begin{array}{l}0.65 \\
0.42\end{array}$ & & & $\begin{array}{l}0.38 \\
0.77\end{array}$ & & & $\begin{array}{l}0.67 \\
0.92\end{array}$ \\
\hline $\begin{array}{l}\text { POMS: } \\
\text { Tension, } t \text {-score } \\
\text { Depression, } t \text { score } \\
\text { Anger, } t \text { score } \\
\text { Fatigue, } t \text { score } \\
\text { Confusion, } t \text { score }\end{array}$ & $\begin{array}{l}0.0039 \\
0.0025 \\
0.0027 \\
0.0036\end{array}$ & $\begin{array}{l}0.0016 \\
0.0011 \\
0.0016 \\
0.0015\end{array}$ & $\begin{array}{l}0.02 \\
0.02 \\
0.09 \\
0.02 \\
0.23\end{array}$ & & & $\begin{array}{l}0.77 \\
0.66 \\
0.67 \\
0.58 \\
0.63\end{array}$ & & & $\begin{array}{l}0.53 \\
0 \cdot 79 \\
0 \cdot 68 \\
0 \cdot 17 \\
0.59\end{array}$ \\
\hline $\begin{array}{l}\text { Pattern memory: } \\
\text { \% Correct } \\
\text { Response time(s) }\end{array}$ & & & $\begin{array}{l}0.64 \\
0.41\end{array}$ & & & $\begin{array}{l}0.71 \\
0.87\end{array}$ & & & $\begin{array}{l}0.82 \\
0.60\end{array}$ \\
\hline $\begin{array}{l}\text { Symbol-digit: } \\
\% \text { Correct } \\
\text { Response time(s) }\end{array}$ & 0.0030 & 0.0011 & $\begin{array}{l}0.32 \\
0.008\end{array}$ & & & $\begin{array}{l}0.59 \\
0.62\end{array}$ & & & $\begin{array}{l}0.95 \\
0.99\end{array}$ \\
\hline
\end{tabular}

*Each line in the table represents a single regression model. Difference in acute exposure, difference in cumulative exposure, and cumulative exposure up to 1988 were included in each model. Covariates included if $\mathrm{p}$ value $<1$. Covariates considered included difference in self report of effort, effect of medication, marijuana use, history of loss of consciousness, history of seizures, history of emotional problems, overtime worked in past week.

tAll differences are 1989 value minus 1988 value.

ĐValue in 1989 minus value in 1988 .

II counterintuitive direction.

Only the symptoms of nausea and inflamed gums were significantly associated with the estimate of cumulative exposure in 1988 and there were only two marginal associations in 1989 (skin dryness and excessive salivation).

\section{Threshold models}

Table 9 compares the association between measures of acute exposure and neuropsychological outcomes for the 1988 cross sectional data in a no threshold model and in models examining possible thresholds at the 75th and 90th percentile of 1988 exposure. Decreased $\mathrm{p}$ values and increased $r^{2}$ values for the threshold models compared with the no threshold models suggest a threshold effect. The test for which the results most strongly suggested a threshold effect was trails A (response time and number of errors), with the 90th percentile showing the strongest associations. Again, the results for trails A errors are explained completely by a single outlier. Although less obvious than the case of trails A, for each of the other tests showing significant associations in the no threshold model (POMS depression, tension, anger, and fatigue), there was some decrease in the $\mathrm{p}$ value and increase in $r^{2}$ for the 75th percentile threshold model. Except for the anger t score, results for the 90th percentile threshold model were less significant on the POMS tests than results for the 75th percentile model. Finally, for the symbol-digit response time, there was a statistically significant asso- ciation only in the 90 th percentile threshold model.

When the same analyses were conducted on the 1989 data (not shown), the only test with a significant association in the no threshold model, symbol-digit response time, showed some increase in significance, particularly in the 90th percentile threshold model. The only other significant association was for visual reproductions immediate recall in the 90th percentile threshold model.

LONGITUDINAL MULTIPLE REGRESSION MODELS Table 10 presents the results of longitudinal regression analyses of the difference in results on neurobehavioural tests in 1988 and 1989 with the differences in acute and cumulative exposure. The differences in test scores were significantly associated with acute exposure in the expected direction for three of the measures of POMS (tension, depression, fatigue) and for symbol-digit response time, with marginal significance for trails A response time and POMS anger. There were no associations in the expected direction with the difference in estimates of cumulative exposure.

\section{Discussion}

The finding in the 1988 cross sectional analyses of a significant association between acute exposure and neuropsychological outcome on trails $A$ and POMS is intriguing and is 
supported by the threshold analyses. Threshold analysis suggests that the daily exposure threshold that best fits the data for trails A performance (a measure of attention and simple sequencing) is $1050 \mathrm{~h} \times \mathrm{mg} / \mathrm{m}^{3}$ whereas a threshold daily exposure of 540 $\mathrm{h} \times \mathrm{mg} / \mathrm{m}^{3}$ fits most of the POMS data best. Few findings emerged from the 1989 data, with only one task (symbol-digit response time) showing a significant association in the no threshold model and an effect at 1050 $\mathrm{h} \times \mathrm{mg} / \mathrm{m}^{3}$ in the threshold model analyses. The 1989 data, however, must be interpreted with caution because mean exposure was significantly lower in 1989 than in 1988 and there were many fewer subjects experiencing higher exposures: only six subjects in 1989 had daily exposures at the level of the 1988 90th percentile $\left(1050 \mathrm{~h} \times \mathrm{mg} / \mathrm{m}^{3}\right)$. The discrepancy in exposure was due to a significant difference in outside temperature between the two years: 1988 was much warmer, necessitating greater use of air conditioning and therefore more recirculation of solvent in plant air. The presence of more symptoms in 1988 than 1989 is also consistent with effects related to higher exposures in the first year.

With regard to the longitudinal data, the finding of statistically significant associations between the difference in acute exposure and the difference in three of the POMS scale scores, as well as borderline significance for the difference in trails $A$ response time and another POMS scale, is also consistent with the occurrence of greater effects related to exposure in 1988. The finding for trails must be interpreted with some caution given the likelihood that practice effects could in themselves improve test performance. Practice effects, however, cannot be invoked to explain differences in POMS. It is also unlikely that subjects consciously or unconsciously deliberately skewed POMS data as they were unaware of the results of measures of personal exposure at the time of neuropsychological testing.

In past studies of neuropsychological effects of solvents, exposure has been shown to be associated with decrements in performance on trails A in subjects exposed to mixtures of solvents ${ }^{27}$ as well as overall trails response and error scores with subjects exposed to carbon disulphide. ${ }^{28}$ In a third study, ${ }^{54}$ trails $\mathrm{A}$ was performed more slowly in workers exposed to toluene and paint solvents than controls, although the difference was not significant. The discrepancy between performance on trails A and trails B in this group was interesting and cannot be directly compared with prior reports. It is likely to reflect the subjects' ability to overcome mild tracking problems and to inhibit impulsive responses when faced with a more challenging task. This explanation is consistent with negative findings on trails $B$ and coupled with negative findings on the Wisconsin card sorting test, another task that challenges tracking capacity.

The POMS subtests seem to be especially sensitive to the effects of solvents at low levels of exposure. We have found a positive association between acute exposure to mixed silk screening solvents and the depression and anger scales on POMS, ${ }^{31}$ and performance on the NES version of the POMS (computerised version of the standard POMS but lacking the vigour scale items) has been found to be associated with exposure to mixed solvents in painters. ${ }^{6869}$

The concurrence of findings of attentional and mood decrements at higher levels of exposure to naphtha is consistent with our prediction that the earliest manifestations of exposure would be in the functional domains of attention and mood. Given this clustering, we do not believe that the positive results from 1988 are due to chance. They are typical of the mild, acute, reversible effects of exposure to solvents described in previous work, ${ }^{47}$ and are consistent with those seen clinically in patients exposed to solvents. ${ }^{4}$ Furthermore, the results support the hypothesis of frontal (as measured by trails) and limbic system (as measured by POMS) dysfunction predicted at the outset of the study.

The strength of the effects found after acute exposure to naphtha at daily concentrations around $540 \mathrm{~h} \times \mathrm{mg} / \mathrm{m}^{3}$ deserves more investigation. Past studies of acute exposure done on humans in exposure chambers have suggested that this exposure level would be too low to produce effects. For example, Gamberale et al concluded that there is a "risk...in workers exposed to $2500 \mathrm{mg} / \mathrm{m}^{3}$ doing light work", 43 and Stokholm showed that exposure to $400 \mathrm{ppm}$ (2100-2400 $\mathrm{mg} / \mathrm{m}^{3}$ ) of white spirits resulted in performance deficits tests. ${ }^{70}$ These conclusions, however, are based on performance measures, not mood, and it seems likely that affective changes are among the earliest manifestations of solvent intoxication. ${ }^{48}$ Also, the occupational exposure situation is significantly different from the experimental setting in that acute and chronic exposures are intertwined and exposure occurs for longer.

The negative findings on effects of cumulative exposure must be interpreted with caution. Whereas they seem to support the conclusion that the effects of exposure to naphtha at this level are only mild, acute, and transitory, the plant had been open only eight years at the time of the study. The opportunity for studying subjects with extensive exposure at a chronic low dose was thus limited. Also, it is notoriously difficult to estimate cumulative lifetime exposures for subjects in a manner that produces strong findings. Anecdotally, the workers in the plant studied were themselves convinced that there was no doubt about the occasional occurrence of acute symptoms after exposure and were concerned about the potential for subacute chronic effects of exposure.

Based on this study, it was recommended that the plant ventilation system be upgraded to limit daily exposure to naphtha to less than $540 \mathrm{mg} / \mathrm{h} / \mathrm{m}^{3}$ (about $90 \mathrm{ppmh}$ ).

The project was wholly supported by joint funds from the United Automobile Workers/General Motors National Joint Committee on Health and Safety. 
1 Waldron HA. Solvents and the brain. Br F Ind Med 1986; 43:73-4.

2 Johnson BL, ed. Prevention of neurotoxic illness in working populations. New York: Wiley, 1987.

3 White RF, Feldman RG, Travers PH. Neurobehaviora effects of toxicity due to metals, solvents and insecticides. Clin Neuropharmacol 1990;13:392-412.

4 White RF, Proctor SP. Solvent encephalopathy. In: Parks RW, Sec RF, Wilson RS, eds. Neuropsychology of Alzheimer's disease and other dementias. New York: Oxford University Press, 1993.

5 Baker AB. The nervous system in trichloroethylene. An experimental study. $\mathcal{F}$ Neuropathol Exp Neurol 1958;17: 649-55.

6 Grandjean E, Munchinger R, Turrian V, Haas PA, Knoepfel HK, Rosenmund H. Investigation into the effects of exposure to trichloroethylene in mechanical engineering. $\mathrm{Br} \mathcal{F}$ Ind $M e d$ 1955;12:131-42.

7 Grandjean E. Trichloroethylene effects on animal behavior. Arch Environ Health 1960;1:106-8.

8 Battig K, Grandjean E. Chronic effects of tricholoroethylene on rat behavior. Arch Environ Health 1963;7: 694-9.

9 Isaacson LG, Taylor DH. Maternal exposure to 1,1,2 tricholoroethylene affects the hippocampal formation of tricholoroethylene affects the hippocampal for

10 Isaacson LG, Spohler SA, Taylor DH. Trichloroethylene affects learning and decreases myelin in the rat hippocampus. Neurotoxicol Teratol 1990;12:375-81.

11 Evans HL, Dempster AM, Snyder CA. Behavioral changes in mice following benzene inhalation. Neuro behavioral Toxicology and Teratology 1981;3:481-5.

12 Stopps GJ, McLaughlin M. Psychological testing of human subjects exposed to solvent vapors. $\mathrm{Am}$ Ind $\mathrm{Hyg}$ Assoc $\mathcal{F}$ 1967;28:43.

13 Salvini $M$. Evaluation of psychophysiological functions in humans exposed to trichloroethylene. $\mathrm{Br} \mathcal{F}$ Ind $\mathrm{Med}$ 1971;28:293-5.

14 Echeverria DE. Acute behavioral effects of toluene and ethanol in humans. Ann Arbor: University of Michigan, 1989. (Dissertation.)

15 Axelson O, Hane M, Hogstedt A. A case referent study of neuropsychiatric disorders among workers exposed to solvents. Scand $\mathcal{F}$ Work Environ Health 1976;2:14-20.

16 Lindstrom $\mathrm{K}$, Harkonen $\mathrm{H}$, Hernberg $S$. Disturbances in psychological functions of workers occupationally exposed to styrene. Scand $\mathcal{f}$ Work Environ Health 1976, 3:129-39.

17 Mikkelson S. A cohort study of disability pension and death among painters with special reference to disabling presenile dementia as an occupational disease. Scand $\mathscr{Y}$ Social Med 1980; (suppl 16):34-43.

18 Olson J, Sabroe S. A case-referent study of neuropsychiatric disorders among workers exposed to solvents in the Danish wood and furniture industry. Scand $\mathcal{f}$ Social Med 1980;(suppl 16):44-9.

19 Rasmussen $H$, Olsen J, Lauritsen J. Risk of encephalopathia among retired solvent-exposed workers. F Occup Med 1985;27:561-6.

20 Brackbill R, Maizlish N, Fishback T. Risk of neuropsychiatric disability among patients in the United States. Scand 7 Work Environ Health 1990;16:182-8.

21 Riise T, Moen BE. A nested case-control study of disability among seamen with special reference to psychiatric disorder and exposure to solvents. Neuroepidemiology 1990;9:88-94.

22 van Vliet C, Swaen G, Volovics A, et al. Neuropsychiatric disorders among solvent-exposed workers. Int Arch Occup Environ Health 1990;62:127-32.

23 Hanninen $H$. Psychological picture of manifest and laten carbon disulphide poisoning. $\mathrm{Br} F$ Ind $\mathrm{Med} 1971 ; 28$ 374-81.

24 Hanninen $H$, Eskelinen L, Husman $K$, Nurminen $M$ Behavioral effects of long-term exposure to a mixture of organic solvents. Scand $\mathcal{f}$ Work Environ Health 1976;2: $240-55$.

25 Hane M, Axelson O, Blume J, Hogstedt C, Sundell L, Ydreborg B. Psychological function changes amon house

26 Elofsson SA, Gamberale F, Hindmarsh T, et al. Exposure to organic solvents: a cross-sectional epidemiologic investigation on occupationally exposed car and industrial spray painters with special reference to the nervous

27 Ryan CM, Morrow LA, Hodgson M. Cacosmia an neurobehavioral dysfunction associated with occupational exposures to mixtures of solvents. Am $\mathcal{F}$ Psychiatry $1988 ; 145: 1442-5$.

28 Matthews CG, Chapman LJ, Woodard AR. Differential neuropsychiatric profiles in idiopathic versus pesticideinduced Parkinsonism. In: Johnson BL, ed. Advances in neurobehavioral toxicology. Chelsea, MI: Lewis Press, 1990:323-30.

29 Gregerson P, Angelso B, Nielsen TE, et al. Neurotoxic effects of organic solvents in exposed workers-an occupational neuropsychological and neurological investigation. Am $\mathcal{f}$ Ind Med 1984;5:201-25.

30 Echeverria DE, White RF, Sampao C. A neurobehavioral evaluation of PCE exposure in patients and dry cleaners: a possible relationship between clinical and preclinical effects. Eighth International Neurotoxicology Conference. Little Rock, AR: 1990.

31 White RF, Feldman RG, Echeverria DE, Schweikert J.
Neuropsychological effects of solvent exposure. Cincinnati: National Institute for Occupational Safety and Health 1990. (Report to NIOSH on grant \#5 K01 OHO 28-03.)

32 Feldman RG, Lessell S. Neuro-ophthalmologic aspects of trichloroethylene intoxication. In: Burnett J, Barbeau A, eds. Progress in neuro-ophthalmology. Amsterdam: Excerpta Medica, 1969: 281-2.

33 Seppalainen AM, Haltia M. Carbon disulfide. In: Spencer PS, Schaumberg $\mathrm{HH}$, eds. Experimental and clinical neurotoxicology. Baltimore: Williams and Wilkins, 1980, 356-73.

34 Fomazzari L, Wilkinson DA, Kapur BM, Carlen PL. Cerebellar and functional impairment in toluene Cerebellar and functional impairment

35 Feldman RG, White RF, Currie JN, Travers PH, Lessell S. Long-term follow-up after single exposure to trichloroethylene. Am $\mathcal{F}$ Ind Med 1985;8:119-26.

36 Stracciari A, Gallasi R, Ciarduli C, Coccagna G. Neuropsychological and EEG evaluation in exposure to trichloroethylene. F Neurol 1985;232:120-2

37 Aaserud O, Gjerstad L, Nakstad P, et al. Neurological examination, computed tomography, cerebral blood flow and neuropsychological examination in workers with long-term exposure to carbon disulfide. Toxicology 1988;49:277-82.

38 Kraut A, Lilis R, Marcus M, Valciukas JA, Wolff MS, Landrigan PJ, Neurotoxic effects of solvent exposure on sewage treatment workers. Arch Environ Health 1988;43 263-8.

39 Peters HA, Levine RL, Matthews CG, Chapman LJ Extrapyramidal and other neurologic manifestation associated with carbon disulfide fumigant exposure. Arch Neurol 1988;25:537-40.

40 Filley CM, Heaton RK, Rosenberg NL. White matter dementia in chronic toluene abuse. Neurology 1990;40: $532-4$

41 Anger WK. Neurobehavioral testing of chemicals: impact on recommended standards. Neurobehavioral Toxicology and Teratology 1984;6:147-53.

42 Hastings L, Cooper GP, Burg W. Human sensory response to selected petroleum hydrocarbons. In: HN MacFarland, et al, eds. Proceedings of the Symposium on the Toxicology of Petroleum Hydrocarbons. Washington, DC: American Petroleum Institute; 1982.

43 Gamberale F, Annwall G, Hultengren M. Exposure to white spirit II. Psychological functions. Scand $\mathcal{f}$ Work Environ Health 1975;1:31-9.

44 Hayhurst E. Poisoning by petroleum distillates. Indian Medical Records 1936;5:53-63. Reported in: National Institute of Occupational Safety and Health. Criteria for a recommended standard occupational exposure to refined petroleum distillates. Cincinnati: Department of Health, petroleum distillates. Cincinnati: Depart

45 White RF. Differential diagnosis of solvent encephalopathy and probable Alzheimer's disease in older workers. Clinical Neuropsychologist 1987;1:153-60.

46 White RF, Feldman RG. Neuropsychological assessment of toxic encephalopathy. Am $\mathcal{F}$ Ind Med 1987;11: 191-6.

47 Baker EL, White RF. Chronic effects of organic solvents on the central nervous system and diagnostic criteria. Copenhagen: World Health Organization, and Oslo: Nordic Council of Ministers. Washington DC: Reprinted by the US Department of Health and Human Services, Public Health Service, 1985.

48 White RF, Feldman RG, Proctor SP. Neurobehavioral effects of toxic exposures. In: White RF ed. Clinical syndromes in adult neuropsychology: the

49 Rocskay AS, Robins TG, Echeverria DE, et al. Estimation of cumulative exposures to naphtha at an automobile fuel-injector manufacturing plant. Am Ind Hyg Assoc $\mathcal{F}$ (in press).

50 American Conference of Governmental Industrial Hygienists. 1991-1992 Threshold limit values for chemical substances and physical agents and biological exposure indices. Cincinnati: ACGIH, 1991.

51 Eller PM, ed. National Institute of Occupational Safety and Health manual of analytical methods. 3rd. ed. Cincinnati: NIOSH, 1984.

52 McNair DM, Lorr M, Droppleman LF. Profile of mood states. San Diego: Educational and Industrial Testing Service, 1971.

53 Reitan RM. Validity of the trail making test as an indicator of organic brain damage. Percep Mot Skills 1958;8: $271-6$

54 Cherry N, Hutchins H, Pace T, Waldron HA Neurobehavioral effects of repeated occupational exposure to toluene and paints. $\mathrm{Br} \mathcal{F}$ Ind $M e d 1985 ; 42$ 291-300.

55 Reitan RM. Theoretical and methodological bases of the Halstead-Reitan neuropsychological test battery. In: Grant I, Adams KM, eds. Neuropsychological assessment of neuropsychiatric disorders. New York: Oxford of neuropsychiatric disor

56 Moss MB, Albert MS, Butters N, Payne M. Differential patterns of memory loss among patients with Alzheimer's disease, Huntington's disease, and alcoholic
Korsakoff's syndrome. Arch Neurol 1986;43:239-46.

57 Wechsler D. The Wechsler memory scale. $\mathcal{F}$ Psychol 1945; 19:87-95.

58 Butters N, Salmon DP, Cullum CM, et al. Differentiation of amnestic and demented patients with the Wechsle 
memory scale revised. Clinical Neuropsychologist 1988;2: 133-48.

59 Letz RE, Baker EL. NES 2: Neurobehavioral evaluation system manual, 1988.

60 Letz RE, Baker EL. Computer-assisted neurobehavioral testing in occupational health. Seminars in Occupational testing in occupational healt

61 Letz R. The neurobehavioral evaluation system: an international effort. In: Johnson B, ed. Advances in neurobe-
havioral toxicology. Chelsea, MI: Lewis Press, 1990, havioral

62 Wechsler D. Wechsler adult intelligence scale-revised manual. New York: Psychological Corporation, 1981.

63 Baker EL, White RF, Murawski B. Clinical evaluation of neurobehavioral effects of occupational exposure to organic solvents and lead. International fournal of Mental Health $1985 ; 14: 135-58$.

64 Berg EA. A simple objective technique for measuring flexibility in thinking using a card sorting method. $f$ Gen Psychol 1948;39:15-22.
65 Lezak MD. Neuropsychological assessment, 2nd ed. New York: Oxford University Press, 1983.

66 Rey A. L'examen psychologique dans les cas d'encephalopathie traumatiques. Archives de Psychologie 1941;28:206-356.

67 SPSS Inc. SPSS- $X$ user's guide, 3rd ed. Chicago: SPSS Inc, 1988

68 Baker EL, Letz RE, Eisen EE, et al. Neurobehavioral effects of solvents in construction workers. $₹$ Occup Med 1988; 30:116-23.

69 Filder AT, Baker EL, Letz R. The neurobehavioral effects of occupational exposure to organic solvents among construction painters, $B r F$ Ind Med 1987;44: 292-308.

70 Stokholm J. Biological effects after short term exposure to aliphatic and aromatic hydrocarbons: report to Sodolin \& Holmblak Ltd, Copenhagen. Summarized in: Grasso $P$, Sharrot M, Davies DM, Irvine D. Neuro psychological and psychological disorders and exposure to organic solvents. Food Chem Toxicol 1984;22:819-52.

\section{NOTICE}

\section{Wellness Forum \\ keeping business healthy}

\section{MAJOR NEW UK AWARD LAUNCHED TO PROMOTE HEALTH AT WORK}

A group of top companies and leading health organisations in the UK are joining together today to launch a major new award to promote the health and wellbeing of working people across the country. The first PPP/ Wellness Forum, Working For Health Award will be presented to the UK company judged to be carrying out the best practices to promote the health and wellbeing of its employees.

Chairman of the Wellness Forum Dr Robert Smith said: "The search is now underway to find the UK company which is doing the best for the health of its employees. With 350 million working days lost in the UK through illness each year, there is a clear incentive for companies to promote the health of their workforce."

The Wellness Forum is a group of some of the best known companies and health organisations in the UK. The founder members are Grand Metropolitan; J Sainsbury; Glaxo Group; The Institute of Personnel Management; and The English National
Board for Nursing, Midwifery and Health Visiting. Other members include Marks and Spencer, IBM, and the Industrial Orthopaedic Society. The Forum meets regularly to develop programmes for improving health in the workplace, to share best practice, and to influence areas for research and training initiatives.

Andrew Randall, General Manager, PPP Corporate Division, said that: "By sponsoring this award, we hope to encourage UK employers to help employees to improve their health. Better morale, lower absenteeism and staff retention are just some of the benefits of wellness programmes."

UK companies who want to win the new PPP/Wellness Forum, Working for Health Award will have to prove that they have a strategy for achieving optimum physical and mental health for their staff and that their programmes for the wellness of employees are established and implemented to the maximum capability of the company. The winner will be announced in April 1994.

For details of how to enter (closing date 15 March 1994), companies should write to Jenny Marshall, Wellness Forum, Priory House, 8 Battersea Park Road, London SW8 4BG. 